\title{
Preparation, Characterization, and Ion Conductivities of the Polymer Electrolytes Based on Poly(ethylene oxide)-g-Poly(ethylene glycol)
}

\author{
Kuk Young CHO, Kyoung-Hee LEE, and Jung-Ki PARK \\ Department of Chemical Engineering, \\ Korea Advanced Institute of Science and Technology, \\ 373-1, Kusung-dong, Yusong-gu, Daejon 305-701, Korea
}

(Received September 22, 1999)

\begin{abstract}
The comb-shaped polyethers, poly(ethylene oxide)-g-poly(ethylene glycol)s, (PEO-g-PEGs) were prepared by condensation polymerization of poly(ethylene glycol) $\left(\bar{M}_{w}=400\right)$ and 3-chloro-2-chloromethyl-1-propene (CCMP) followed by a graft reaction with poly(ethylene glycol) methylether, and their ionic conduction behavior was investigated. The ionic conductivities of the PEO-g-PEG complexed with $\mathrm{LiCF}_{3} \mathrm{SO}_{3}$ were investigated by varing the side chain length of the graft polymers and also the salt concentration. The maximum ion conductivity obtained from the graft polymer-based polymer electrolytes was $4.0 \times 10^{-5} \mathrm{~S} \mathrm{~cm}^{-1}$ at $25^{\circ} \mathrm{C}$. The shift of the optimum [EO]/[LiCF $\left.\mathrm{SO}_{3}\right]$ for maximum ion conductivity to a higher value compared to pristine and conventional linear PEO was observed, and it seemed to be due to the enhanced salt solvation imparted by the graft structure of PEO-g-PEG. The crosslinking of PEO-g-PEG by a free radical reaction could provide the improved mechanical property of the polymer electrolytes.
\end{abstract}

KEY WORDS Polymer Electrolyte / Solvomercuration-Demercuration / Ionic Conductivity / Salt Solvation / Poly(ethylene oxide) /

Solid polymer electrolyte (SPE) has attracted a great deal of attention in the field of electrochemistry owing to its potential application to an electrolyte material for energy storage rechargeable batteries. ${ }^{1}$ There are several advantages in the application of the SPE to rechargeable batteries compared to liquid electrolyte such as absence of electrolyte leakage or gassing which ensures safety of the battery, light weight, and capability of operating over a wide temperature range. ${ }^{2,3}$ Among the numerous candidates for the solid polymer electrolyte, poly(ethylene oxide) (PEO) has been most extensively studied as a typical matrix polymer of solvent-free polymer electrolytes.

However, there is a considerable drawback to be overcome for practical application of the SPE to rechargeable batteries. The ion conductivity of the PEO/alkali metal complexes has not yet reached the required value ( $10^{-4} \mathrm{~S} \mathrm{~cm}^{-1}$ ) due to the high degree of crystallinity inherent in the complexes at room temperature. ${ }^{4}$ Various attempts such as the block copolymerization, ${ }^{5,6}$ hyperbranching, ${ }^{7,8}$ formation of interpenetrating network, ${ }^{9}$ and grafting ${ }^{10-14}$ for PEO have thus been made to reduce the crystallinity of the PEO based polymer electrolytes. Many studies of grafting on PEO have been focused on the introduction of oxyethylene unit onto the non-ion solvating flexible main chains of which repeating units are methacrylate, ${ }^{10}$ itaconate, ${ }^{11}$ or polyphosphazene. $^{12}$

Recently, studies on the polymer electrolytes based on glycidylether which can generate the ion solvating unit at the side chain were reported. ${ }^{13,14}$ They studied on the crosslinked system in which the number of oxyethylene units in the side chain was rather small. They reported that the high ionic conductivity, using lithium bis(trifluoromethanesulfonyl) imide(LiTFSI) as a salt of the

\footnotetext{
${ }^{\dagger}$ To whom all correspondence should be addressed.
}

polymer electrolyte, was induced by the fast motion of short oligo(oxyethylene) side chains in their graft polymers. However, there is still a need to consider further the structural variation of PEO-g-PEG by changing the number of oxyethylene units in the side chain to a greater extent and to investigate their ion conduction behavior. In order to do this, we have used a different synthesis method for PEO-g-PEG that can produce a much longer oxyethylene side chain from the previous work.

In this work, PEOs containing the PEG units in the side chain were synthesized by condensation reaction, and the ion conduction behavior of the polymer electrolytes based on the grafted PEOs was studied. The effect of PEG side chain length and the salt concentration on the ion conductivities were also investigated.

\section{EXPERIMENTAL}

\section{Preparation of LPC 400 and PEO-g-PEG}

Figure 1 shows the reaction scheme for the synthesis of linear polycondensate and PEO-g-PEG. The linear polycondensate was prepared by polycondensation of $\alpha$, $\omega$-dihydroxy oligo(oxyethylene) (PEG, $\bar{M}_{w}=400$ ) and $3-$ chloro-2-chloromethyl-1-propene (CCMP). Finely grounded $\operatorname{KOH}(10.8 \mathrm{~g})$, chlolobenzene $(250 \mathrm{~mL})$ and $\operatorname{PEG}\left(\bar{M}_{w}\right.$ $=400)(16 \mathrm{~g})$ were mixed at $40^{\circ} \mathrm{C}$ under nitrogen atmosphere. CCMP ( $5 \mathrm{~g}$ ) in chlorobenzene $(50 \mathrm{~mL})$ was then added to the previous mixture. The resulting solution was stirred under nitrogen for $22 \mathrm{~h}$. After hooding chlorobenzene for a day, acetonitrile which is a non-solvent for potassium salt was added, and the insoluble inorganic products such as $\mathrm{KCl}$ and $\mathrm{KOH}$ were removed by centrifuging at $15000 \mathrm{rpm}$ for $1 \mathrm{~h}$. The solvent was evaporated in a hood for a day and then the polymer product was thoroughly dried under vacuum for two days. The obtained linear polymer based on PEG of molecular weight 400 was designated as the linear polycondensate 400 


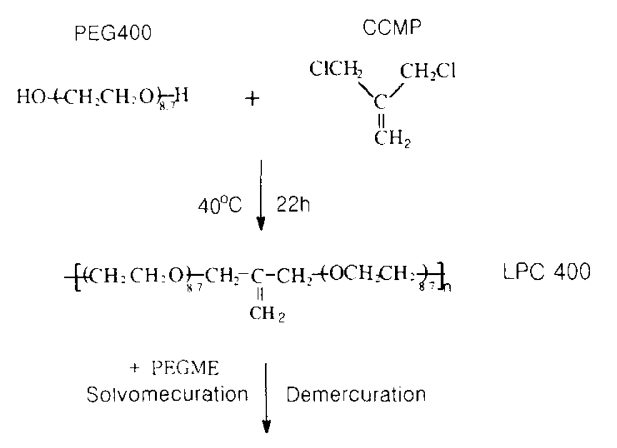

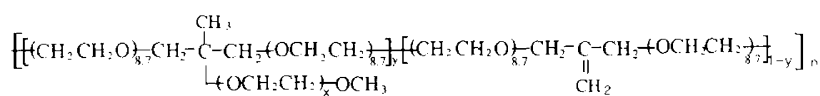

$$
\begin{aligned}
& \text { PEO-g-PEG } \\
& y=0.6 \quad x=2,7.2,11,8,16.3
\end{aligned}
$$

Figure 1. Reaction scheme for the synthesis of LPC 400 and PEO-g-PEG.

\section{(LPC 400) in this article.}

The PEO-g-PEG was synthesized by solvomercuration and demercuration reaction $(S-D$ reaction) of the $L P C$ 400 and poly(ethylene glycol) methyl ether (PEGME). PEGME $\left(M_{w}=120,350,550,750\right)$ was dried under vacuum for a day at $60^{\circ} \mathrm{C}$ prior to the reaction. The LPC 400 $(2.5 \mathrm{~g})$ was dissolved in $100 \mathrm{~mL}$ of tetrahydrofuran and then PEGME was added to the LPC 400 solution. Mercury trifluoroacetate, $\mathrm{Hg}\left(\mathrm{CF}_{3} \mathrm{COO}\right)_{2}(2.44 \mathrm{~g})$, was added for solvomercuration before sealing the flask with stopper. The mixture was then agitated for one day. Sodium borohydride $\left(\mathrm{NaBH}_{4}\right)(0.72 \mathrm{~g})$ was slowly added to the solvomercurated mixture and agitated for about two days until the solution became transparent and mercury drop appeared. After mercury was removed, the polymer product was precipitated with diethylether. Then it was purified to remove the inorganic salt by centrifuging the product solution. Finally, the polymer product was washed again by diethylether three times followed by drying under vacuum for two days. The obtained PEO-gPEG was designated in this paper as graft $x$, where $x$ represents the molecular weight of the PEG side chain.

\section{Network Formation}

Cross-linking of PEO-g-PEG was induced by free radical reaction of the graft polymer using dibenzoyl peroxide (BPO) as an initiator in an acetonitrile solution. After concentration of the solution at a temperature of 45 ${ }^{\circ} \mathrm{C}$ under dry nitrogen, the solutions were heated to $70^{\circ} \mathrm{C}$ for $5 \mathrm{~h}$ to proceed the free radical reaction. The obtained network films were dried under nitrogen atmosphere for two days and then vacuum dried for another two days.

\section{Preparation of Polymer Electrolytes}

The appropriate amount of PEO-g-PEG and $\mathrm{LiCF}_{3} \mathrm{SO}_{3}$ were mixed in acetonitrile, and heated to $60^{\circ} \mathrm{C}$ until they were completely dissolved in the solvent. The solutions were then placed on a Teflon plate and the solvent was evaporated under dry nitrogen atmosphere. The residual solvent was completely removed by drying in vacuum for several days.

\section{${ }^{l}$ H Nuclear Magnetic Resonance (NMR) Measurement}

The ${ }^{1} \mathrm{H}$ NMR experiments were performed on a Bruker-AMX-500 NMR spectrometer. The polymer film dissolved in deuteriated chloroform $\left(\mathrm{CDCl}_{3}\right)$ was transferred into a NMR tube, subsequently sealed to prevent access of humidity to the sample.

\section{Thermal Analysis}

The differential scanning calorimetry (DSC) studies were carried out to determine the glass transition and melting temperatures of the samples using the Du Pont 2000 thermal analyzer instrument. Samples were loaded in hermetically-sealed cells and measurements were taken over a temperature range -120 to $150^{\circ} \mathrm{C}$ at a heating rate of $10^{\circ} \mathrm{C} \mathrm{min}^{-1}$ under nitrogen atmosphere. The recorded $T_{\mathrm{g}}$ was taken as the inflection point and $T_{\mathrm{m}}$ was given as the peak temperature of the melting endotherm.

\section{Conductivity Measurement}

Polymer electrolyte films were sandwiched between the two stainless steel electrodes ( $12 \mathrm{~mm}$ in diameter). The ionic conductivities of the polymer electrolyte film was measured by complex impedance analysis using a Solatron 1255 frequency response analyzer coupled to an IBM PS/2 computer over the frequency range of $100 \mathrm{~Hz}$ $-10 \mathrm{MHz}$. The real and imaginary parts of the complex impedance were plotted, and the ionic conductivity $(\sigma)$ could be obtained from the bulk resistance $\left(R_{\mathrm{b}}\right)$ found in complex impedance diagram.

\section{Infrared Measurement}

Films for infrared measurements were prepared by spreading the acetonitrile solution over $\mathrm{Krs}-5$ window. After acetonitrile was evaporated under atmosphere for one day, the samples were dried at $40^{\circ} \mathrm{C}$ for more than one day under vacuum to completely remove the residual solvent. The FT-IR spectra were recorded in the absorption mode on Bomem MB 102 FT-IR spectrometer at a resolution of $4 \mathrm{~cm}^{-1}$. Minimums of 40 scans were signal-averaged and the spectra were stored on a magnetic disc system for further analysis.

\section{RESULTS AND DISCUSSION}

\section{Structure Characterization of LPC 400 and PEO-g-PEG}

The structural analyses of these materials were performed by using the ${ }^{1} \mathrm{H}$ NMR spectra of the materials. Figure 2 shows the ${ }^{1} \mathrm{H}$ NMR spectra of the LPC 400(Figure $2 \mathrm{a}$ ) and the graft 350 (Figure $2 \mathrm{~b}$ ). The detailed peak assignments are given in this figure. These peak assignments were based on the spectra of the pure PEG $\left(\bar{M}_{w}=\right.$ 400), CCMP, and poly(ethylene glycol) methyl ether. The peaks at $\delta=3.57-3.80 \mathrm{ppm}$ correspond to methylene protons in the internal oxyethylene units. The methylene proton adjacent to the double bonds appeared at $\delta=$ $4.10 \mathrm{ppm}$ and the proton of the methylene unit at the double bonds resonated further down field at $\delta=5.20$ ppm. The doublet, which appeared at $5.30 \mathrm{ppm}$, was found to be from the end group protons. The number average molecular weight $\left(M_{n}\right)$ of the synthesized linear polymer(LPC 400) could be calculated from the relative peak area ratios of the proton at $5.3 \mathrm{ppm}$ to the protons which are attached to the double bond. The number av- 


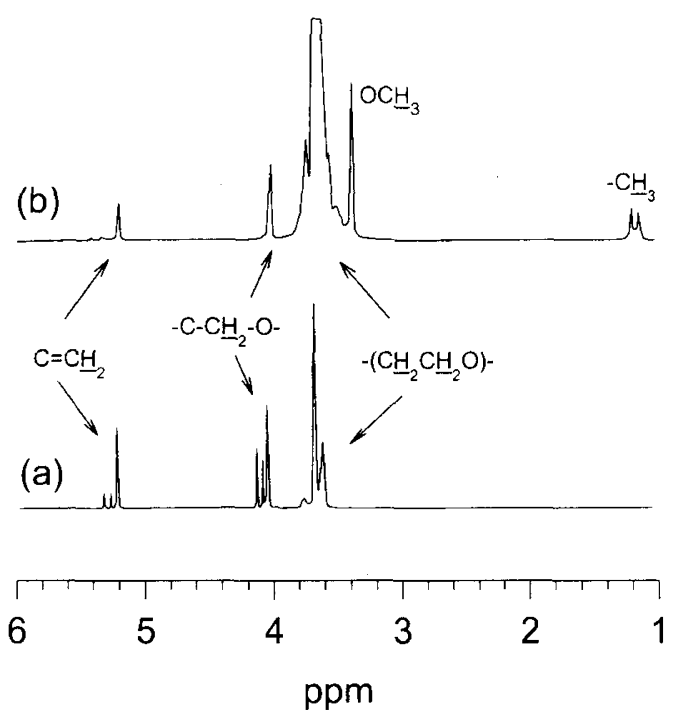

Figure 2. ${ }^{1} \mathrm{H}$ NMR(500 MHz) spectra of (a)PEO-g-PEG(for graft 350 with $60 \%$ graft degree) and (b)LPC 400 in $\mathrm{CDCl}_{3}$ at $25^{\circ} \mathrm{C}$.

erage molecular weight $\left(\vec{M}_{n}\right)$ of the LPC 400 was 6300 . For the graft polymer (graft 350), two new peaks have appeared as shown in Figure 2a. One was from the protons of the methyl group (1.2 ppm) bonded directly to the backbone of the LPC 400 and the other corresponds to the methoxy protons at the end of the grafted PEGME $(3.4 \mathrm{ppm})$. The peaks for the- $\mathrm{OH}$ unit were not found in the spectra for the graft 350 , indicating that the unreacted PEGME was completely removed. The graft degree of the graft polymers could be calculated from the peak areas of the methylene protons of the double bond $(5.2 \mathrm{ppm})$ before and after the graft reaction.

\section{Thermal Characterization of LPC 400 and PEO-g-PEG}

The thermal behavior of the LPC 400, and PEO-g-PEG without containing lithium salt was investigated by DSC measurements. The DSC thermograms for the LPC 400 and its graft polymers are typically shown in Figure 3 and their results are summarized in Table I. The glass transition temperature of the LPC 400 is found to be $-55^{\circ} \mathrm{C}$. When the short PEG side chain was grafted to the main chain (graft 120), there was a little increase in $T_{\mathrm{g}}$ compared to the LPC 400 . It seems to be due to the hindered rotation of the main chain caused by the newly introduced methyl group from the CCMP through S-D reaction. As the side chain length increased further (graft 350 ), the side chain flexibility could induce larger free volume generation leading to the decrease in $T_{\mathrm{g}}$ of the graft polymer. However, with the further increase of the side chain length (PEG side chain molecular weight $>350$ ), the $T_{\mathrm{g}}$ of the graft polymer increased again. It indicates that there is an optimum length of the side chain for maximum mobility of the chains, above which the introduction of further ethylene oxide segments in the side chain provides chain packing between the side chains and consequently reduces the chain mobility, which is consistent with the results of others who worked with non-ion solvating main chain with larger variation of PEG side chains. ${ }^{10-12}$

An endothermic peak due to the melting of the crystalline of the LPC 400 was observed at $4^{\circ} \mathrm{C}$ and the degree

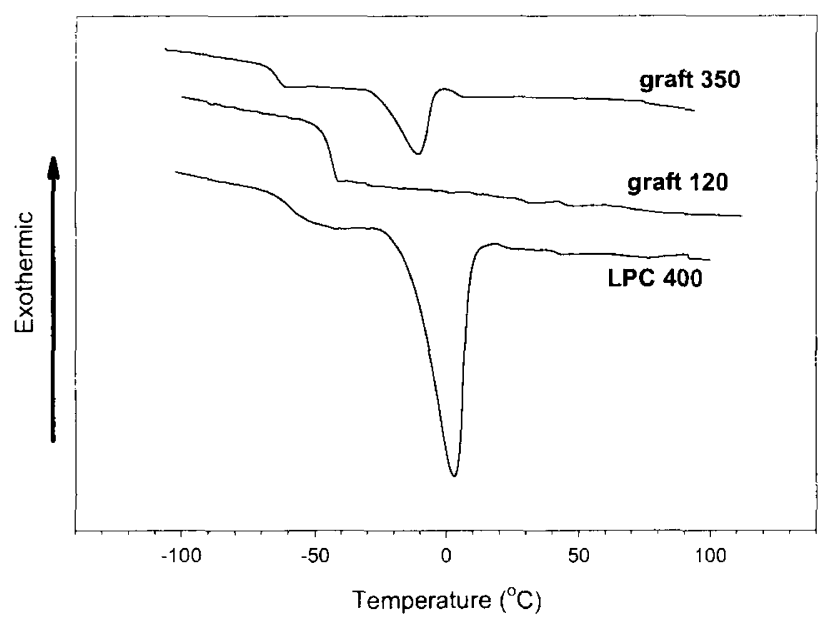

Figure 3. DSC thermograms of the LPC 400 and the graft polymers.

Table I. DSC results of LPC 400 and PEO-g-PEGs

\begin{tabular}{lrrr}
\hline Samples & $T_{\mathrm{g}}{ }^{\circ} \mathrm{C}$ & $T_{\mathrm{m}}{ }^{\circ} \mathrm{C}$ & $\Delta H_{\mathrm{m}} / \mathrm{J} \mathrm{g}^{-1}$ \\
\hline LPC 400 & -55 & 4 & 52.5 \\
Graft 120 & -44 & - & - \\
Graft 350 & -63 & -11 & 28.1 \\
Graft 550 & -56 & 21 & 75.9 \\
Graft 750 & -42 & 35 & 89 \\
\hline
\end{tabular}

of crystallinity, which was estimated from the ratio of the experimentally determined $\Delta H_{\mathrm{m}}$ to the $\Delta H_{\mathrm{m}}$ value of $203 \mathrm{~J} \mathrm{~g}^{-1}$ for the perfectly crystalline PEO, ${ }^{15}$ was $25.9 \%$. In the case of the graft polymer(the graft 120), however, it showed no melting transition. This indicates that the side chain of the graft 120 is too short to form the crystalline phase. As the side chain length is further increased, the melting transition appeared again and the melting temperature together with $\Delta H_{\mathrm{m}}$ of the graft polymer were increased. This melting transition is considered to be generated by the packing of the side chains for the following two reasons. One is that the melting temperatures of the graft polymers and the corresponding poly(ethylene glycol) methylethers were almost same. The other reason is that there is no trace of melting peak for the graft polymers near $4{ }^{\circ} \mathrm{C}$ which is the melting temperature of the LPC 400.

\section{Side Chain Length Effect on Ion Conductivity}

Figure 4 shows the ionic conductivities of the polymer electrolytes based on the graft polymers with the graft degree of $60 \%$ as a function of molecular weight of the side chain in the temperature range of $15-65^{\circ} \mathrm{C}$. The salt concentration $\left([\mathrm{EO}] /\left[\mathrm{LiCF}_{3} \mathrm{SO}_{3}\right]\right)$ for all the polymer electrolytes were fixed at 15 . The optimum molecular weight of the side chain for maximum ion conductivity at room temperature was found to be 350 , above which the ion conductivities were decreased due to the reduction in the chain mobility which is one of the most important factors influencing the ion conductivity. The chain mobility can be easily evaluated by the glass transition temperature of the polymer electrolytes.

Table II summarized the DSC results of the polymer electrolytes based on PEO-g-PEG. All the polymer electrolytes based on these graft polymers showed no melt- 


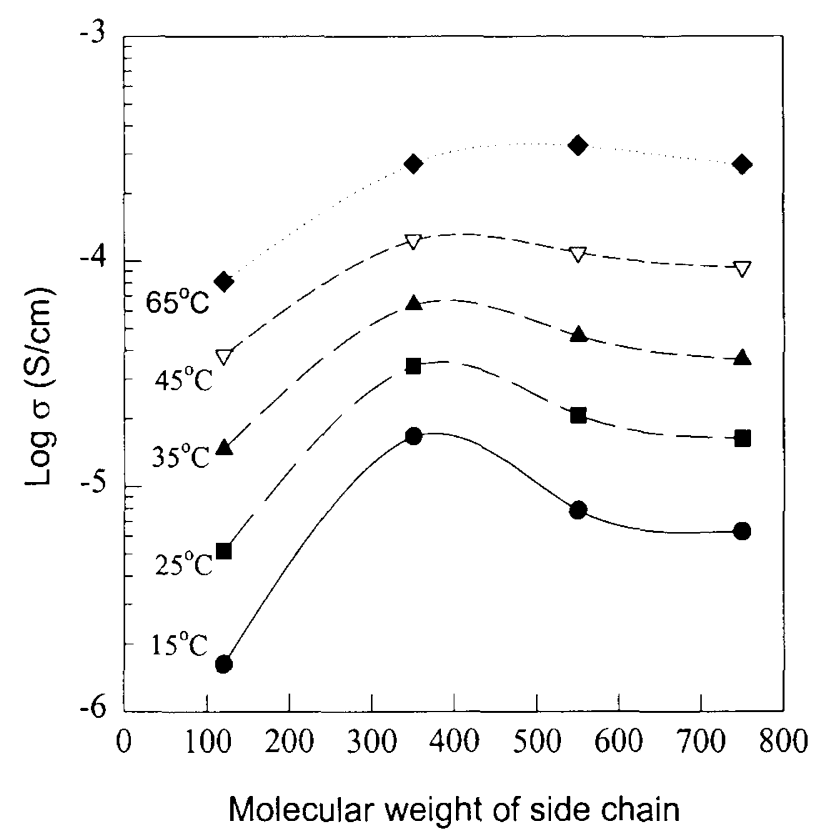

Figure 4. Ionic conductivities of the PEO-g-PEG based polymer electrolytes as a function of side chain length.

Table II. DSC results of the polymer electrolytes based on $\mathrm{PEO}-\mathrm{g}-\mathrm{PEG}$ and $\mathrm{LiCF}_{3} \mathrm{SO}_{3}$

\begin{tabular}{cccc}
\hline Samples & $T_{\mathrm{g}} /{ }^{\circ} \mathrm{C}$ & $T_{\mathrm{m}} /{ }^{\circ} \mathrm{C}$ & $\Delta T_{\mathrm{g}} /{ }^{\circ} \mathrm{C}^{\mathrm{a}}$ \\
\hline Graft 120 & -27 & - & 17 \\
Graft 350 & -42 & - & 21 \\
Graft 550 & -38 & - & 18 \\
Graft 750 & -30 & - & 12 \\
\hline & & & $\left([\mathrm{EO}] /\left[\mathrm{LiCF}_{3} \mathrm{SO}_{3}\right]=15\right)$
\end{tabular}

${ }^{\mathrm{a}} \Delta T_{\mathrm{g}}=T_{\mathrm{g}}$ of polymer with salt minus $T_{\mathrm{g}}$ of polymer without salt.

ing transition, which indicates that all the lithium complexed graft polymers are completely amorphous when the salt is introduced with the salt concentration of [EO]/ $\left[\mathrm{LiCF}_{3} \mathrm{SO}_{3}\right]=15$. When the salt is added to the matrix polymer, a decrease of chain flexibility, evidenced by the increase in the glass transition temperature, is generally observed. ${ }^{16}$ It is due to the additional physical crosslinking arising from the interactions of cations with the solvating groups of the polymer matrix. The difference in the $T_{\mathrm{g}}$ of the polymer electrolyte and the $T_{\mathrm{g}}$ of the matrix polymer without containing the $\operatorname{salt}\left(\Delta T_{\mathrm{g}}\right)$ can represent the degree of interaction between the lithium cations and the solvating groups of the matrix polymer, ${ }^{17}$ and this can be related to the number of charge carriers which is another important factor on the ionic conductivities. The graft 350 showed the highest $\Delta T_{\mathrm{g}}$ value, which may represent the graft 350 has the largest number of charge carriers among the polymer electrolytes studied in this work.

It is also found from Table II that the polymer electrolyte based on the graft 350 has the lowest glass transition temperature among the polymer electrolytes studied, which indicates that the graft 350 has the highest chain segmental mobility. The highest mobility and $\Delta T_{\mathrm{g}}$ of the graft 350 could produce the highest ion conductivity at room temperature.

\section{Salt Concentration Effect on Ion Conductivity}

The ionic conductivities of the polymer electrolytes
Table III. DSC results of the polymer electrolytes based on

\begin{tabular}{|c|c|c|c|c|c|}
\hline \multicolumn{6}{|c|}{$\mathrm{LPC} 400 / \mathrm{LiCF}_{3} \mathrm{SO}_{3}$ and graft $550 / \mathrm{LiCF}_{3} \mathrm{SO}_{3}$} \\
\hline$[\mathrm{EO}] /\left[\mathrm{LiCF}_{3} \mathrm{SO}_{3}\right]$ & $T_{g} /{ }^{\circ} \mathrm{C}$ & $T_{\mathrm{m}} /{ }^{\circ} \mathrm{C}$ & {$[\mathrm{EO}] /\left[\mathrm{LiCF}_{3} \mathrm{SO}_{3}\right]$} & $T_{\mathrm{g}} /{ }^{\circ} \mathrm{C}$ & $T_{\mathrm{m}} /{ }^{\circ} \mathrm{C}$ \\
\hline $5 / 1$ & -27.8 & - & $10 / 1$ & -36.5 & - \\
\hline $10 / 1$ & -36.8 & - & $25 / 1$ & -50.4 & - \\
\hline $15 / 1$ & -45.3 & - & $50 / 1$ & -53.3 & - \\
\hline $25 / 1$ & -51.2 & -1.6 & $75 / 1$ & -54.2 & - \\
\hline $50 / 1$ & -54.5 & 3.0 & $100 / 1$ & -55.5 & 21.8 \\
\hline
\end{tabular}

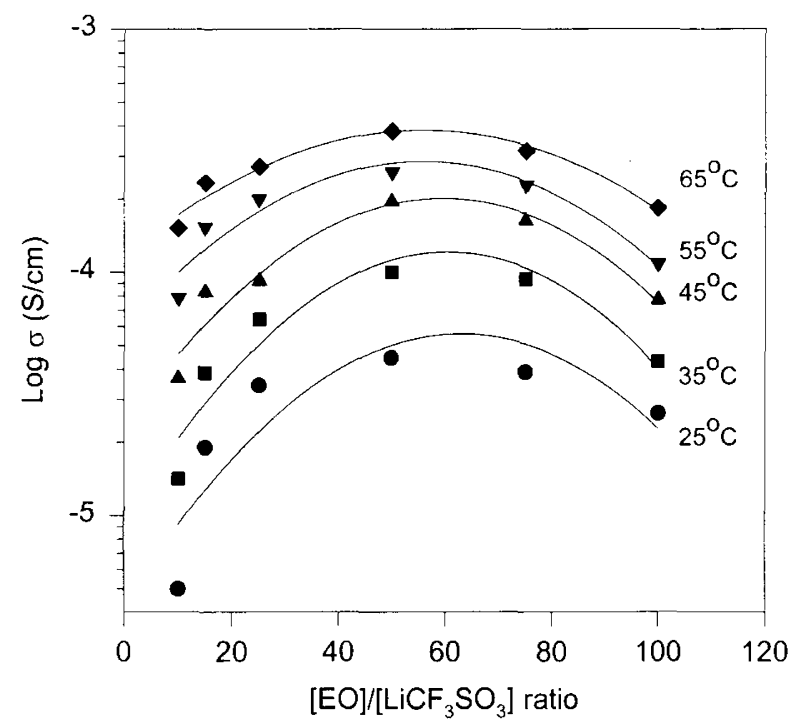

Figure 5. Ionic conductivities of the graft $550(80 \%$ graft degree) based-polymer electrolytes as a function of salt concentration.

with various graft degrees and side chain lengths as a function of the salt concentration, $[\mathrm{EO}] /\left[\mathrm{LiCF}_{3} \mathrm{SO}_{3}\right]$, was also investigated. The measurements of ion conductivities were carried out by cooling the samples from $65^{\circ} \mathrm{C}$ to room temperature followed by heating to the predetermined temperatures for ion conductivity measurement in order to maintain good contact between the electrolyte and the blocking electrodes. Table III represents the DSC results of the polymer electrolytes based on LPC $400 / \mathrm{LiCF}_{3} \mathrm{SO}_{3}$ and graft $550 / \mathrm{LiCF}_{3} \mathrm{SO}_{3}$. It is found that the polymer electrolytes based on the graft polymer could be amorphous even at the lower salt concentration of $[\mathrm{EO}] /\left[\mathrm{LiCF}_{3} \mathrm{SO}_{3}\right]=75$, while those based on the LPC 400 become amorphous at the higher salt concentration of $[\mathrm{EO}] /\left[\mathrm{LiCF}_{3} \mathrm{SO}_{3}\right]=15$. This may be due to the much looser crystalline structure induced by the side chain of graft polymer in the polymer electrolyte based on the graft polymer.

The ionic conductivity isotherms of the polymer electrolytes based on the graft 550 as a function of salt concentration are shown in Figure 5. It shows that the ionic conductivities initially increase, reach maximum and then fall off with salt concentration. The lower ionic conductivities observed at higher salt concentration is a consequence of higher $T_{\mathrm{g}}$ of the polymer electrolyte as shown in Table III. The lower ion conductivity at lower salt concentration is due to the insufficient amount of charge carrier. The optimum salt concentration for maximum ion conductivity could thus be found at [EO]/ 


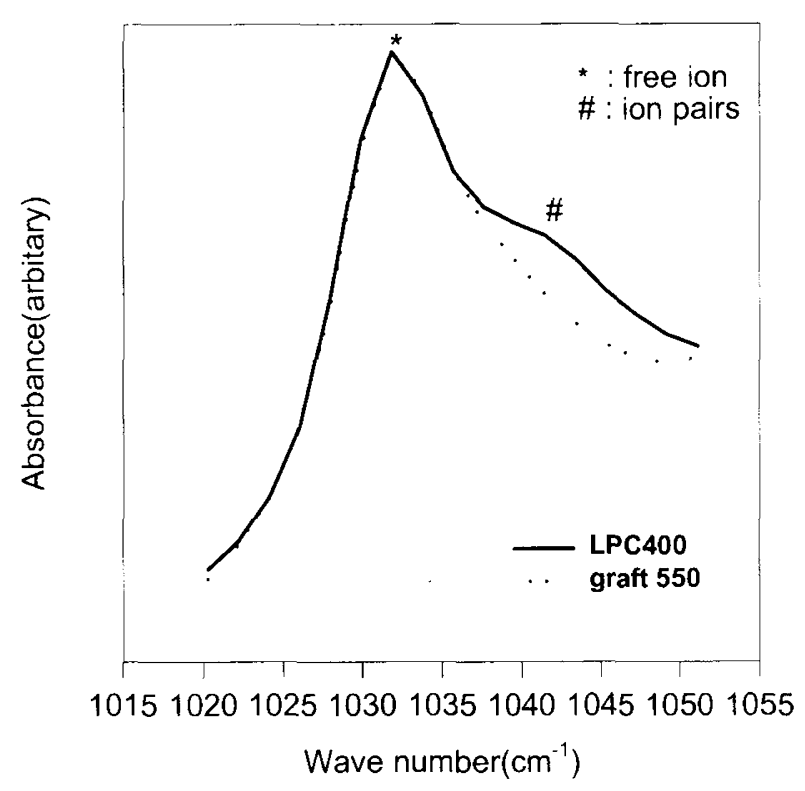

Figure 6. FT-IR spectra for the symmetric stretching vibration of $\mathrm{CF}_{3} \mathrm{SO}_{3}{ }^{-}$anion for $\mathrm{LPC} 400 / \mathrm{LiCF}_{3} \mathrm{SO}_{3}$ and graft $550 / \mathrm{LiCF}_{3} \mathrm{SO}_{3}$ $\left([\mathrm{EO}] /\left[\mathrm{Li}^{+}\right]=50\right)$.

\section{$\left[\mathrm{LiCF}_{3} \mathrm{SO}_{3}\right]=50$.}

This salt concentration for the maximum ionic conductivity for the polymer electrolyte based on the graft polymer is significantly different from those observed from the LPC based polymer electrolytes. The LPC 400 based polymer electrolyte showed the maximum ion conductivity around $[\mathrm{EO}] /\left[\mathrm{LiCF}_{3} \mathrm{SO}_{3}\right]=20$. The differences in the chain structures between the linear PEO(LPC 400) and PEO-g-PEG are thought to be the reason for the shift of the value of $[\mathrm{EO}] /\left[\mathrm{LiCF}_{3} \mathrm{SO}_{3}\right]$ for maximum ion conductivity of the polymer electrolyte based on PEO-g-PEG to a higher value. Because of the high mobility of the side chain and comb-shaped structure of the matrix polymer for the polymer electrolyte based on PEO-g-PEG, it is expected that the graft polymer showed a higher solvation ability than the LPC 400 based polymer electrolyte. An enhanced solvation ability can be evidenced by measuring the number of charge carriers for the above two kinds of polymer electrolytes. The number of charge carrier can be estimated from the relative area of the peaks corresponding to the free ions and ion pairs in the FT-IR spectra. Figure 6 shows the superposed IR spectra of the polymer electrolytes based on the LPC 400 and the graft 550 in the range of $1020-1050 \mathrm{~cm}^{-1}$. The peaks at 1032 $\mathrm{cm}^{-1}$ and $1041 \mathrm{~cm}^{-1}$ were assigned to the stretching vibration of the free anion, $\mathrm{CF}_{3} \mathrm{SO}_{3}{ }^{-}$, and ion pairs respectively. The assignment was referenced from other's work. ${ }^{19-21}$ It is found that the graft 550 based polymer electrolyte has less relative amount of ion pairs than the one based on the LPC 400 . Since the graft 550 has more end groups than LPC 400, free volume of the graft 550 would be larger than that of LPC 400 . It is thus expected that the graft 550 is more advantageous in forming a proper conformation for complexing with a salt. The relative fraction of the free ion for each polymer electrolyte was obtained by the peak resolution of the FT-IR spectra for the free ion and ion pair peaks and the absolute amount of the free ion in the polymer electrolyte was calculated by multiplying the amount of the added

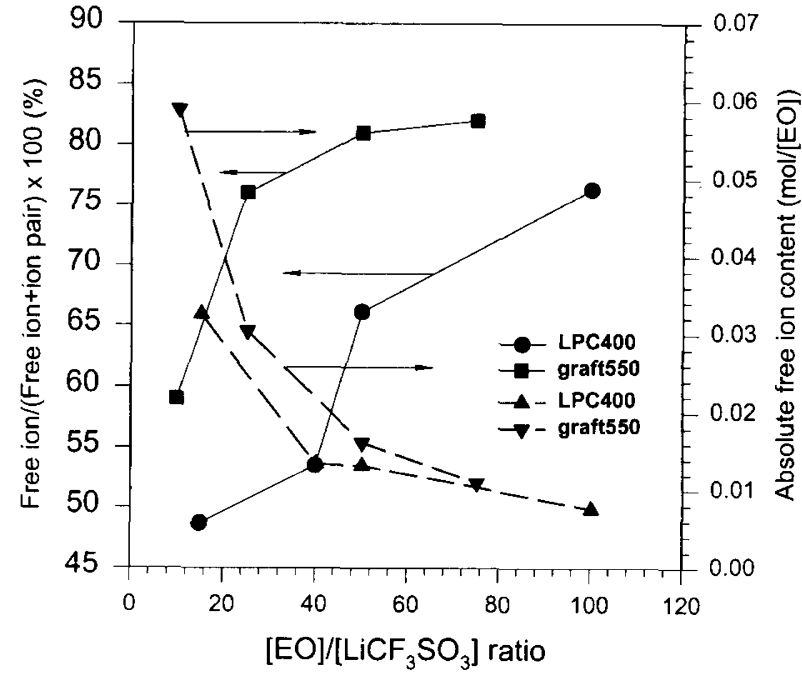

Figure 7. The relative fraction of free ions and the absolute amount of free ions for $\mathrm{LPC} 400 / \mathrm{LiCF}_{3} \mathrm{SO}_{3}$ and graft $550 / \mathrm{LiCF}_{3} \mathrm{SO}_{3}$ with salt concentration.

salt by the relative fraction of the free ion, and the results are shown in Figure 7 . For both kind of polymer electrolytes, the relative fraction of the free ion, which is represented by a solid line, is shown to increase as the salt concentration decreases, and the absolute free ion content, which is shown by a dotted line, decreases with the decrease of the salt concentration. The increase in the free ion fraction and the decrease in the absolute amount of the free ions with the increase of the ratio, $[\mathrm{EO}] /\left[\mathrm{LiCF}_{3} \mathrm{SO}_{3}\right]$, is in good agreement with the results of the previous works. ${ }^{22}$

Enhanced solvating ability of the graft 550-based polymer electrolytes compared to the LPC 400-based ones could thus make sufficient number of charge carrier at small amount of salt introduction. This results in less amount of $\operatorname{salt}\left([\mathrm{EO}] /\left[\mathrm{Li}^{+}\right]=50\right)$ required for maximum ion conductivity of the graft 550 -based polymer electrolyte than the LPC 400.

\section{Network Formation of PEO-g-PEO}

For practical application of the PEO-g-PEG based polymer electrolytes to high energy density batteries, mechanical properties(free standing film) of the polymer electrolytes should be importantly considered. Crosslinking of the matrix polymer is the well-known method to obtain the dimensional stability of polymer electrolytes. The cross-linking of PEO-g-PEG at the site of residual double bond in the CCMP unit was thus proceeded after S-D reaction. The completion of the crosslinking reaction was evidenced by the disappearance of the residual double bond in CCMP in ${ }^{1} \mathrm{H}$ NMR spectra. Table IV lists the representative ion conductivities of the crosslinked and the non-crosslinked PEO-g-PEG based polymer electrolytes. The BPO content used for the cross-linking reaction was $10 \mathrm{~mol} \%$ of the amount of double bond in LPC 400 . Even if the polymer chain flexibility can be restricted by the cross-linking, there was only a little decrease in the ionic conductivity for the crosslinked PEO-g-PEG based polymer electrolyte com- 
Table IV. The ion conductivities of the graft 350-based polymer electrolytes with and without crosslinking reaction at $25^{\circ} \mathrm{C}$

\begin{tabular}{|c|c|c|}
\hline & $\begin{array}{l}\text { Without cross-linking } \\
\text { reaction }\end{array}$ & $\begin{array}{l}\text { With cross-linking } \\
\text { reaction }\end{array}$ \\
\hline Ion conductivities $/ \mathrm{S} \mathrm{cm}^{-1}$ & $14.02 \times 10^{-5}$ & $1.93 \times 10^{-5}$ \\
\hline Film mechanical state & Gel state & Free standing \\
\hline
\end{tabular}

pared to the non-crosslinked PEO-g-PEG based polymer electrolyte. The free standing film of the polymer electrolyte could be successfully obtained through the crosslinking of PEO-g-PEG.

\section{CONCLUSION}

PEO-g-PEG that has a wide range of oligo(oxyethylene) side chain length was successfully synthesized by the condensation reaction followed by the S-D reaction. It was found that there is an optimum length of the oligo(oxyethylene) side chain for maximum ion conductivity of the polymer electrolytes based on the graft polymers. It was also interestingly found that the amount of salt for maximum ion conductivity of the polymer electrolytes based on the graft polymer was much smaller than that in the conventional PEO based polymer electrolytes. This seems to be due to the enhanced lithium saltsolvating power of the newly grafted PEO-g-PEG.

The crosslinking of PEO-g-PEG could provide the mechanical stability of the polymer electrolyte without any significant sacrifice in the ionic conductivity.

Acknowledgment. This work has been supported by the Korea Ministry of Science and Technology in 1997.

\section{REFERENCES}

1. D. E. Fenton, J. M. Parker, and P. V. Wright, Polymer, 14, 589 (1973).

2. P. G. Bruce, Ed., "Solid state electrochemistry", Cambridge University Press, New York, N. Y., 1995, Chapter 5.

3. J. Owen, in "Compresive polymer science", Vol. 2, Sir G. Allen and J. C. Bevington, Ed., Pergamon, Oxford, 1989, p 669.

4. J. R. MacCallum and C. A. Vincent, Ed., "Polymer electrolyte review I", Elsevier, London, 1987.

5. C. Robitalle and J. Prud'homme, Macromolecules, 19, 987 (1986).

6. J. Przyluski and Wieczorek, Solid state Ionics, 53, 1071 (1992).

7. E. Cloutet, J. L. Fillaut, Y. Gananou, and D. J. Astruc, J. Chem. Soc., Chem Commun., 2433 (1984).

8. M. Gauthier and M. Moller, Macromolecules, 24, 4548 (1991).

9. C. K. Chiang, B. L. Bauer, R. M. Briber, and G. T. Davis, Polymer Commun., 28, 34 (1982).

10. D. W. Xia, D. Soltz, and J. Smid, Solid State Ionics, 14, 221 (1984).

11. J. M. G. Cowie and A. C. S. Martin, Polymer Commun., 26, 298 (1985).

12. P. M. Blonsky, D. F Shriver, P. Austin, and H. R. Allock, J. Am. Chem. Soc., 106, 6854 (1984).

13. K. Motogami, M. Kono, S. Mori, M. Watanabe, and N. Ogata, Electrochimica Acta, 37, 1725 (1992).

14. S. Kohjiya, Y. Ikeda, and Y. Matoba, The Sixth International Symposium on Polymer Electrolytes, p 4, Kanagawa, Japan (1998).

15. B. Wuderlich, "Macromolecular Physics"., Vol. 3, Academic Press, New York, N. Y., 1980.

16. M. A. Ratner and D. F. Shriver, Chem. Rev., 88, 109 (1988).

17. D. W. Kim, J. K. Park, H. W. Rhee, and H. D. Kim, Polym. J., 26, 993 (1994).

18. X. Peng, S. Wu, and D. Chen, Solid State Ionics, 59, 197 (1993).

19. I. Albinsson, P. Jacobsson, B-E. Mellander, and J. R. Stevens, Solid State Ionics, 53-56, 1044 (1992).

20. M. Kakihana, S. Schantz, and L. M. Torell, J. Chem. Phys., 92, 6271 (1990).

21. I. Albinsson, B-E. Mellander, and J. R. Stevens, Solid State Ionics, 63 (1993).

22. B. Sandner, J. TÜbke, S. Wartewig, and S. Shashkov, Solid State Ionics, 83, 87 (1996). 